\title{
A NOTE ON ANALYTIC SELF-MAPPINGS
}

\author{
By TAKao Kato and Yoshinisa Kubota
}

\section{Dedicated to Professor Yukinari Tôki on his 60th birthday}

1. Statements and notations. Recently, Marden, Richards and Rodin [7] studied analytic self-mappings of Riemann surfaces and proved a number of theorems related to homotopy some of which involve Huber's results [4]. Jenkins and Suita [5] also investigated this theory from the other point of view. In their paper they gave an alternative proof of the result in [7]. As a counterpart of it they proved a similar result related to homology only for plane regions. In the present paper we shall extend their result to Riemann surfaces.

Throughout this paper if nothing otherwise is indicated we denote by $W$ a Riemann surface whose fundamental group is non-abelian, and by $\hat{W}$ the KerékjártóStoilow compactification of $W . H_{1}(W)$ denotes the 1-dimensional homology group of $W$. Let $f$ be an analytic self-mapping of $W$. It is known that $f$ induces an endomorphism $E_{f}$ by the mapping $c \rightarrow f(c)$ for $c \in H_{1}(W)$. Let $f^{n}$ denote the $n$-th iteration of $f$. Then our main theorem is stated as follows:

THEOREM. Let $H$ be a non-trivial subgroup of $H_{1}(W)$ with finite rank. Suppose that the restriction of the induced homomorphism $E_{f} \mid H$ is an endomorphism. If the kernel of the restriction $E_{f} \mid H$ is trivial, $f$ is either an automorphism of finite period or $\left\{f^{n}\right\}$ tends to an isolated ideal boundary component of harmonic dimension one uniformly on every compact subset of $W$. In the latter case H reduces to an infinite cyclic group generated by a dividing cycle. Furthermore, if the ideal boundary component is a non-planar boundary then $E_{f} \mid H$ reduces to the identity mapping.

This theorem is also an extension of a theorem of Komatu and Mori [6]. The authors express their heartiest thanks to Professor N. Suita for his encouragement in preparing this paper.

2. Lemmas. It is convenient to give some preparatory lemmas. The following lemma related to the iteration of analytic self-mappings is found in Heins [3].

Lemma 1. Suppose that $K_{1}$ and $K_{2}$ are given compact subsets of $W$. If $f$ neither possesses a fixed point nor has finite period, then $f^{n}\left(K_{1}\right)$ lies in one and the same component of $W-K_{2}$ for $n$ sufficiently large. If $f$ has a fixed point and is

Recelved April 25, 1972. 
not an automorphism then for any neighborhood $N$ of the fixed point, $f^{n}\left(K_{1}\right) \subset N$ for $n$ sufficiently large.

The following topological lemma is a counterpart of lemma 5 of Jenkins and Suita [5].

LEMMA 2. Let $S$ be a canonical subregion of $W$. Suppose that there exist connected components $\Sigma_{\imath}(i=1,2)$ of $\hat{W}-S$. If there exist cycles $c_{i}(i=1,2)$ in $\Sigma_{\imath} \cap W$ respectively, satisfying $c_{1} \sim c_{2}+0$ (reads $c_{1}$ is homologous to $c_{2}$ and not homologous to zero), then $\hat{W}-\left(W \cup \Sigma_{1} \cup \Sigma_{2}\right)=\phi$; i.e. $W-\Sigma_{1}-\Sigma_{2}$ is of finite genus and with two boundary components.

Proof. At first we assume that $c_{1}$ is a nondividing cycle in $W$. Then so is it in $\Sigma_{1} \cap W$. Hence, there exists a differential $\omega$ in $\Gamma_{c 0}\left(\Sigma_{1} \cap W\right)$ such that $\int_{c_{1}} \omega \neq 0$ where as to $\Gamma_{c 0}\left(\Sigma_{1} \cap W\right)$ the reader will be referred to Ahlfors and Sario [1]. If we set $\omega^{\prime}=\omega$ in $\Sigma_{1} \cap W, \omega^{\prime}=0$ in $W-\Sigma_{1}$ we have $\omega^{\prime} \in \Gamma_{c}(W)$. This implies $\int_{c_{1}-c_{2}} \omega^{\prime}$ $=\int_{c_{1}} \omega \neq 0$, which contradicts the fact $c_{1} \sim c_{2}$. Therefore $c_{1}$ and $c_{2}$ must be dividing cycles.

Assume that $\hat{W}-\left(W \cup \Sigma_{1} \cup \Sigma_{2}\right) \neq \phi$. There exists a canonical subregion $T$ of $W$ such that $c_{1} \cup c_{2} \cup S \subset T$ and $\partial T \cap\left(W-\Sigma_{1}-\Sigma_{2}\right) \neq \phi$. We can write $\partial T=\cup_{\jmath=1}^{n} \gamma_{\jmath}$, where $\gamma_{j}(j=1, \cdots, n)$ are all the components of $\partial T$. Furthermore we may assume $\gamma_{j} \subset W$ $-\Sigma_{1}-\Sigma_{2}$ for $j=1, \cdots, l, \gamma_{j} \subset \Sigma_{1}$ for $j=l+1, \cdots, m$ and $\gamma_{j} \subset \Sigma_{2}$ for $j=m+1, \cdots, n$ where $0<l<m<n$. Giving appropriate orientations to $\gamma_{\jmath}(j=1, \cdots, n)$ we take $\gamma_{j}(j=2, \cdots, n)$ as a part of homology basis of $W$. Then there exist integers $a_{j}(j=l+1, \cdots, n)$ satisfying $c_{1} \sim \sum_{j=l+1}^{m} a_{j \gamma_{\jmath}}, c_{2} \sim \sum_{j=m+1}^{n} a_{j} \gamma_{\jmath}$. This contradicts the fact $c_{1} \sim c_{2}$.

In the following three lemmas we treat the boundary behavior of a special analytic mapping.

LEMMA 3. Let $\alpha$ be an isolated ideal boundary component of $W$. Let $c$ be a dividing cycle which divides $\alpha$ from all the other ideal boundary components of $W$. If $f(c) \sim m c(c \nsim 0, m \neq 0)$ and $\left\{f^{n}(c)\right\}$ tends to $\alpha$ uniformly, then there exists a neighborhood $N$ of $\alpha$ such that $f(N) \subset N$. Furthermore $f(N)$ is also a neighborhood of $\alpha$.

Proof. Let $N$ be a neighborhood of $\alpha$. If $f(N) \nsubseteq N$ we shall consider two cases, that is whether $f(N)-N$ is compact or not.

In the former case we may assume $f(N) \cap N \neq \phi$. By lemma 1 there is an $n_{0}$ such that

$$
f^{n}(f(N)-N) \subset N
$$

for all $n \geqq n_{0}$. Furthermore

$$
f^{n+1}(N)-f^{n}(N) \subset f^{n}(f(N)-N) .
$$

Therefore, we have 


$$
M=N \smile \bigcup_{n=0}^{\infty}\left(f^{n+1}(N)-f^{n}(N)\right)=\bigcup_{n=0}^{\infty} f^{n}(N)=\bigcup_{n=0}^{n_{0}+1} f^{n}(N)
$$

where $f^{0}$ means the identity mapping. Then $M$ is a neighborhood of $\alpha$ and $f(M)$ $\subset M$. The homological condition, lemma 1 and the fact $f(M) \subset M$ imply that $f(M)$ is a neighborhood of $\alpha$. This is a desired neighborhood.

If the latter case occurs we let $N_{1}$ be a noncompact component of $f(N)-N$ and $N_{1}^{\prime}$ a noncompact component of the inverse image of $N_{1}$. Then there exists a neighborhood $M$ of $\alpha$ satisfying $M \subset N$ and $N_{1}^{\prime}-M \neq \phi$. From lemma 1 we have

$$
f^{n}(c) \subset M
$$

for $n$ sufficiently large. On the other hand, since $f(c) \sim m c$ we have

$$
N_{1} \cap f^{n+1}(c) \supset f\left(f^{n}(c) \cap N_{1}^{\prime}\right) \neq \phi .
$$

Then we have

$$
N_{1} \cap f^{n+1}(c) \nsubseteq M
$$

This is a contradiction.

In consequence of lemma 3 we have

LEMMA 4. $\alpha, c$ and $f$ are as in lemma 3. If a sequence $\left\{p_{n}\right\}$ of points in $W$ tends to $\alpha$, then the sequence $\left\{f\left(p_{n}\right)\right\}$ also tends to $\alpha$.

Proof. It is sufficient to show the existence of a difining sequence $\left\{N_{i}\right\}$ of $\alpha$ such that $N_{\imath+1} \subset f\left(N_{i}\right) \subset N_{i}$ and $\partial N_{i+1} \subset N_{i}$ for each $i$. Let $N_{1}$ be a neighborhood in lemma 3. We take another neighborhood $N^{\prime}$ of $\alpha$ such that $N^{\prime} \subset f\left(N_{1}\right)$ and $\partial N^{\prime} \subset f\left(N_{1}\right)$. If $f\left(N^{\prime}\right) \subset N^{\prime}$ we take $N_{2}=N^{\prime}$. If $f\left(N^{\prime}\right) \oplus N^{\prime}$ we take $N_{2}=\cup_{n=0}^{\infty} f^{n}\left(N^{\prime}\right)$. Then we have $N_{2} \subset f\left(N_{1}\right)$ and $\partial N_{2} \subset N_{1}$. Succesively we can take $N_{3}, N_{4}, \cdots$.

From lemma 3 and lemma 4 we have

Lemma 5. Suppose that $\alpha, c, f$ and $m$ are as in lemma 3 . Then there exists a neighborhood $N$ of $\alpha$ such that $f(N)$ is included in $N$ and the restriction of $f$ to $N$ is a $|m|$-to-one mapping of $N$ onto $f(N)$.

Proof. Let $M$ be a neighborhood of $\alpha$ satisfying $f(M) \subset M, f \mid M$ the restriction of $f$ to $M$ and $\nu_{f \mid M}(q)$ the valence function of $f \mid M$. By lemma 3 and lemma 4 we can choose a neighborhood $M^{\prime}$ of $\alpha$ such that $f\left(M^{\prime}\right) \subset M^{\prime} \subset f(M)$ and $f(\partial M)$ is included in the noncompact component of $W-f\left(M^{\prime}\right)$. For simlicity's sake we denote $f\left(M^{\prime}\right)$ by $M_{1}$. By lemma 4 we have $1 \leqq \nu_{f \mid M}(q)<\infty$ for every $q$ in $M_{1}$. Fix a point $q_{0}$ of $M_{1}$ and let $E$ be the set of all the points $q$ in $M_{1}$ satisfying $\nu_{f \mid M}(q)=\nu_{f \mid M}\left(q_{0}\right)$. Evidently $E$ is nonempty. We shall prove that $E$ is both open and closed as a subset of $M_{1}$.

First we show that $E$ is open. If $q$ is in $E$, there are points $p_{1}, \cdots, p_{r}$ in $M$ 
such that $f\left(p_{i}\right)=q$ for $i=1, \cdots, r$ and the total multiplicities sum to $\nu_{f \mid M}\left(q_{0}\right)$. There are disjoint neighborhoods $U_{i}$ of $p_{i}$ such that $f$ maps each $U_{i}$ exactly $k_{i}$-to-one onto a neighborhood of $q$, where $k_{i}$ denotes the multiplicity of $f$ at $p_{i}$. Therefore, for each point $q^{\prime}$ in $V=\bigcap_{i=1}^{r} f\left(U_{i}\right)$ we have $\nu_{f \mid M}\left(q^{\prime}\right) \geqq \nu_{f \mid M}\left(q_{0}\right)$. If $E$ is not open we may suppose that there is a sequence $\left\{q_{n}\right\}$ of points in $V$ which converges to $q$ such that $q_{n}=f\left(p_{n}^{\prime}\right)$ for some $p_{n}^{\prime}$ in $M-\cup_{i=1}^{r} U_{i}$. By lemma $4\left\{p_{n}^{\prime}\right\}$ has an accumulation point $p_{0}$ in $M-\cup_{\imath=1}^{r} U_{\imath}$. Therefore, we can find a subsequence $\left\{p_{n_{j}}^{\prime}\right\}$ which converges to $p_{0}$. Clearly $p_{0}$ cannot be any of $p_{i}, i=1, \cdots, r$. On the other hand

$$
f\left(p_{0}\right)=\lim _{\jmath \rightarrow \infty} f\left(p_{n_{j}}^{\prime}\right)=q \text {. }
$$

This is a contradiction.

Next we show that $E$ is closed. Let $\left\{q_{n}\right\}$ be a sequence of points in $E$ which converges to a point $q$ in $M_{1}$. By the above argument there is a neighborhood of $q$ on which $\nu_{f \mid M}$ is equal to $\nu_{f \mid M}(q)$. Since this neighborhood contains $q_{n}$ for sufficiently large $n$ we have $\nu_{f \mid M}(q)=\nu_{f \mid M}\left(q_{0}\right)$.

Hence we conclude that $E=M_{1}$. Let $N_{1}$ be the inverse image of $M_{1}$ with respect to $f \mid M$. Then $f\left(N_{1}\right) \subset N_{1}$ and $f$ maps $N_{1} k$-to-one onto $f\left(N_{1}\right)$ for a positive integer $k$. Let $M_{2}$ be the complement of the noncompact component of $W-M_{1}$ and let $N$ be the inverse image of $M_{2}$ with respect to $f \mid M$. Then $N$ coincides with the complement of the noncompact component of $W-N_{1}$. Therefore we have $f(N) \subset N$ and $f$ maps $N k$-to-one onto $f(N)$. Since $W-N$ and $W-f(N)$ are both connected, we have $\partial N \sim \partial f(N) \sim c$ and $f(\partial N) \sim m \partial f(N)$ where the orientations of $\partial N$ and $\partial f(N)$ are given appropriately. Hence we conclude that $k$ is equal to $|m|$. Thus $N$ is our desired neighborhood.

We remark that we can take our neighborhood $N$ in the preceding lemma so that $W-N$ and $W-f(N)$ are connected and $\partial f(N)$ is contained in $N$. The connectedness has been proved in the lemma. We shall show the latter. Suppose that the conclusion fails for two neighborhood $N_{1}$ and $N_{2}$ in lemma 5 such that $W-N_{i}$ and $W-f\left(N_{i}\right)(i=1,2)$ are connected and $\partial N_{2} \subset f\left(N_{1}\right)$. Then $f$ is a $|m|$-to-one mapping of $N_{1}-\bar{N}_{2}$ onto $f\left(N_{1}\right)-\overline{f\left(N_{2}\right)}$. Let $\mu$ be the harmonic measure of $\partial f\left(N_{1}\right)$ with respect to $f\left(N_{1}\right)-\overline{f\left(N_{2}\right)}$. Since $\mu \circ f$ is the harmonic measure of $\partial N_{1}$ with respect to $N_{1}-\bar{N}_{2}$, we have $\mu \circ f-\mu \leqq 0$ in $f\left(N_{1}\right)-\bar{N}_{2}$. Therefore, $\{p \mid \mu \circ f(p)<1 / 2\} \cup \bar{N}_{2}$ is a desired neighborhood. Considering the direction of $c$ we know that $m$ is a positive integer.

3. Proof of theorem. During the discussion of this paragraph we suppose that $f$ is not an automorphism of finite period. From lemma 1, since the kernel of $E_{f} \mid H$ is trivial, $f^{n}$ tends to an ideal boundary component of $W$ for every compact subset of $W$.

First we assume that there exists a cycle $c(+0)$ satisfying $f(c) \sim m c$ for an integer $m \neq 0$. Let $\left\{R_{n}\right\}$ be a canonical exhaustion of $W$ such that $c \subset R_{0}$. For $n^{\prime}>n$ each component of $R_{n^{\prime}}-\bar{R}_{n}$ is a canonical subregion of $W$. Then there exist $n_{1}, m_{1}$ such that $f^{n_{1}}(c)$ is contained in a component of $R_{m_{1}}-\bar{R}_{0}$ by lemma 1 . We denote this component by $S_{1}$. Next we take $n_{2}, m_{2}$ such that $n_{2}>n_{1}, m_{2}>m_{1}$ and 
$f^{n_{2}}(c)$ is contained in a component of $R_{m_{2}}-\bar{R}_{m_{1}}$ which we denote by $S_{2}$. Evidently we may assume that $S_{2}$ is contained in the component of $W-\bar{R}_{0}$ which contains $S_{1}$. Successively, we take $n_{3}<n_{4}<\cdots, m_{3}<m_{4}<\cdots$ and $S_{3}, S_{4}, \cdots$. Since $f^{n}(c) \sim m^{n} c$ for all $n$, by lemma $2 S_{i}$ is of finite genus and with two boundary components for each $i$. Therefore, $\cup_{i=1}^{k} \bar{S}_{\imath}$ is of finite genus and with two boundary components. From this we conclude that for every compact subset of $W, f^{n}$ tends to the isolated ideal boundary component $\alpha$ which is determined by a defining sequence $\left\{\Delta_{k}\right\}$ where $\Delta_{k}=\cup_{\imath=k}^{\infty} \bar{S}_{\imath}$.

Secondly, we shall consider the general case. Let $\left\{c_{i}\right\}$ be a system of generators of $H$ and let $R$ be a canonical subregion of $W$ which contains this system. Then we have

$$
f^{n}(R) \subset W-R
$$

for sufficiently large $n$. For every $c$ in $H, f^{n}(c)$ is homologous to some linear combination of the cycles of $H$ which are contained in $R$. This shows that $f^{n}(c)$ is homologous to a multiple of a dividing cycle in $H$ by the same reason as in the first step of the proof of lemma 2.

To prove the rest of theorem we take a sequence $\left\{N_{n}\right\}$ of neighborhoods of $\alpha$ such that $W-N_{n}, W-f\left(N_{n}\right)$ are connected, $\partial N_{n+1} \subset f\left(N_{n}\right), \partial f\left(N_{n}\right) \subset N_{n}, f\left(N_{n}\right)-\bar{N}_{n+1}$ is connected and $f$ maps $N_{n}$ exactly $m$-to-one onto $f\left(N_{n}\right)$ for each $n$, where $m$ is a positive integer. The existence of this sequence is guaranteed by lemma 5 and the following remark. Let $t_{n}$ denote the number of the relative boundary components of $N_{n}$ and $s_{n}$ the number of the relative boundary components of $f\left(N_{n}\right)$. Since we cannot know whether $N_{n}-\overline{f\left(N_{n}\right)}$ is connected or not, we denote the number of the connected components of $N_{n}-\overline{f\left(N_{n}\right)}$ by $k_{n}$ and the total sum of the genera of these components by $g_{n}$. Let $h_{n}$ denote the genus of $f\left(N_{n}\right)-\bar{N}_{n+1}$. Since $f$ is an $m$-to-one mapping of $N_{n}-\bar{N}_{n+1}$ onto $f\left(N_{n}\right)-\overline{f\left(N_{n+1}\right)}$, from the RiemannHurwitz relation we have

$$
\begin{aligned}
& 2\left(g_{n}+h_{n}+s_{n}-k_{n}\right)+t_{n}+t_{n+1}-2 \\
\geqq & m\left(2\left(g_{n+1}+h_{n}+t_{n+1}-k_{n+1}\right)+s_{n}+s_{n+1}-2\right) .
\end{aligned}
$$

Since $m s_{n} \geqq t_{n} \geqq s_{n} \geqq k_{n} \geqq 1$, if $m>1$ we have

$$
g_{n}-1+s_{n}-k_{n}>m\left(g_{n+1}-1+s_{n+1}-k_{n+1}\right) .
$$

Therefore, there is an $n_{0}$ such that

$$
g_{n}-1+s_{n}-k_{n}=-1
$$

for all $n \geqq n_{0}$. Since $g_{n} \geqq 0$ and $s_{n} \geqq k_{n}$ we have $g_{n}=0$ and $s_{n}=k_{n}$. By (1) if $g_{n}=0$ and $s_{n}=k_{n}$ we have

$$
t_{n+1}-2 \geqq m\left(t_{n+1}-2\right) .
$$


Here, equality occurs only for $t_{n+1}=s_{n+1}=2$. In this case from (1) and (2) we have

$$
t_{n+2} \geqq 2 m t_{n+2}-m s_{n+2} \geqq m t_{n+2}
$$

which is a contradiction. Therefore, there is an $n_{1}$ such that $g_{n}=0$ and $t_{n}=s_{n}$ $=k_{n}=1$ for all $n \geqq n_{1}$. Then we have easily $h_{n}=0$ for all $n \geqq n_{1}$.

Thus $f^{n+1}(c)$ is freely homotopic to $m f^{n}(c)$ for sufficiently large $n$ provided $m>1$. Hence we conclude that $\alpha$ is an isolated pointlike boundary component (cf. $[5,7])$. Therefore $\alpha$ is trivially of harmonic dimension one.

If $m=1, f$ maps $N$ one-to-one onto $f(N)$. Then by the discussion of Komatu and Mori [6] which uses a criterion of Heins [2], we conclude that $\alpha$ is of harmonic dimension one. This completes the proof.

\section{Remarks.}

REMARK 1. The conclusion in lemma 5 is merely the local property: Let $W=\{0<|z|<1\}-\{8 / 9\}, c=\{|z|=1 / 2\}$ and

$$
f(z)=\left(\frac{(z+1)^{3}-1}{9}\right)^{m} \quad(m>0, \text { an integer }) .
$$

Then $f(c) \sim m c$ and $f^{n}(c)$ tends to $z=0$ uniformly, but $f$ is not an $m$-to-one mapping globaly.

REMARK 2. Huber [4] and Marden, Richards and Rodin [7] showed that if $f(c)$ is freely homotopic to $-c$ then $f^{2}$ is the identity mapping. But the following example shows that this fact does not hold for the homological condition: Let $W$ be the Riemann surface of genus one and with four boundary components defined by the equation

$$
y^{2}=x^{4}-1, \quad r<|x|<1 / r \quad(0<r<1) .
$$

Let $c$ be a cycle which consists of two closed curves corresponding $|x|=r^{\prime}\left(r<r^{\prime}<1\right)$, where the orientation of two curves are the same in the $x$-plane. Let $f$ be a conformal mapping of $W$ onto itself defined by

$$
f:(x, y) \rightarrow\left(\frac{1}{x}, \frac{i y}{x^{2}}\right)
$$

Then $f(c) \sim-c$ but $f^{2}$ is not the identity mapping.

\section{REFERENCES}

[1] Ahlfors, L. V., And L. SArio, Riemann surfaces. Princeton Univ. Press. Princeton (1960).

[2] Heins, M., Riemann surfaces of infinite genus. Ann. of Math. 55 (1952), 296-317. 
[ 3 ] Heins, M., On a problem of Heinz Hopf. J. Math. pures Appl. 37 (1958), 153-160.

[4] Huber, H., Über analytische Abbildungen Riemannscher Flächen in sich. Comment. Math. Helv. 27 (1953), 1-73.

[5] Jenkins, J. A., ANd N. Suita, On analytic self-mappings of Riemann surfaces. to appear in Math. Ann.

[6] Komatu, Y., AND A. Mori, Conformal rigıdity of Riemann surfaces. J. Math. Soc. Japan 4 (1952), 302-309.

[7] Marden, A., I. Richards, And B. Rodin, Analytic self-mappings of Riemann surfaces. J. Analyse Math. 18 (1967) 197-225.

Department of Mathematics,

Tokyo Institute of Technology, and

ToKyo Gakugei University. 